\author{
Associate Professor Mihaela COVRIG, PhD \\ E-mail: mihaela.covrig@ csie.ase.ro \\ The Bucharest University of Economic Studies \\ Associate Professor Paul TĂNĂSESCU, PhD \\ E-mail: paul.tanasescu@fin.ase.ro \\ The Bucharest University of Economic Studies \\ Lecturer Iulian MIRCEA, PhD \\ E-mail: iulian.mircea@csie.ase.ro \\ The Bucharest University of Economic Studies
}

\title{
FUZZY INFORMATION AGGREGATION IN INSURANCE
}

\begin{abstract}
The uncertainty faced by insurance companies may include various aspects, such as the possibility of partial withdrawal in a life insurance which leads to a liquidity risk, or health care benefits claims. Acting in a competitive market is another source of uncertainty as the insurer characteristics may be essential in being preferred by a possible client. Thus, in real-life, there are many situations in which the information cannot be assessed precisely in numerical values. Fuzzy phenomena exist in nature and are encountered within human society. The notion of a fuzzy set has been introduced by L. A. Zadeh in 1965 and has been since developed in many directions. As well, researchers and practitioners have identified applications in many fields. In this paper, we assume that the characteristics of the insurance products with respect to the attribute are represented by intuitionistic fuzzy numbers. The purpose of this paper is to develop an aggregation procedure based on a weighted intuitionistic fuzzy Bonferroni mean and an aggregation procedure introduced by Xu and Yager in 2011, for the multi-attribute decision making and selection of the insurance product.

Key words:fuzzy number,fuzzy set, intuitionistic fuzzy set, intuitionistic fuzzy Bonferroni mean, generalized fuzzy aggregation operator.
\end{abstract}

\section{JEL Classification:C020, G230, G320.}

\section{Introduction}

Fuzzy phenomena exist in nature and are encountered within human society. In real-life, there are many situations in which the information cannot be assessed precisely in numerical values.

Fuzzy sets and fuzzy logic are used for modeling imprecise modes of reasoning that play an essential role in the remarkable human ability to make rational decisions in an environment of uncertainty and imprecision. In the real

DOI: 10.24818/18423264/52.4.18.03 
world applications, membership functions are usually formed based on by the user's intuition. The notion of a fuzzy set has been introduced by L. A. Zadeh in 1965 in order to formalize concepts, in connection with the representation of human natural language and computing with words. A fuzzy set can be defined mathematically by assigning to each possible individual in the universe of discourse a value representing its grade of membership in the fuzzy set. Atanassov (1986) introduced the concept of intuitionistic fuzzy set as a generalization of fuzzy set. He added in the definition of the fuzzy set a new component which determines the degree of non-membership.

The Universal Life policy is a complex product for the insurer. The flexibility granted to the policyholder involves many risks. Thus, it is difficult to predict future profits, due to the uncertainty on the premium level. The possibility of partial withdrawal determines a liquidity risk, therefore it is difficult to match the liabilities with appropriate assets, and so on. The product can be very attractive. The insurer can try to gain the loyalty of the policyholder by means of designing an insurance package which provides capital protection and other insurance benefits during the working life of the insured, and then pension benefits after retirement. Health insurance benefits can also be included in policy: accident insurance, disability benefits, hospitalization benefits, and so on.

Recently, some researchers have proposed many aggregation operators for multiple attribute decision making problems. Torra (2010) introduced the concept of hesitant fuzzy set, which is a generalization of the concept of fuzzy set. He studied the relationship with intuitionistic fuzzy set, and proved that the envelope of the hesitant fuzzy sets is an intuitionistic fuzzy set. Xia and Xu (2011) developed some operators for aggregating hesitant fuzzy information, such as hesitant fuzzy weighted averaging operator, hesitant fuzzy weighted geometric operator, generalized hesitant fuzzy weighted averaging operator, etc. $\mathrm{Xu}$ and Yager (2011) developed some operators for aggregating intuitionistic fuzzy information, such as weighted intuitionistic fuzzy Bonferroni mean operator. Chen et al. (2013) introduced a new type of fuzzy preference structure, called intervalvalued hesitant preference relation, and they gave systematic aggregation operators to aggregate interval-valued hesitant fuzzy information. Miheț and Zaharia (2014) gave a generalization of the notion of fuzzy normed space. Gong and Hai (2016) derived some further properties of convex and quasi-convex fuzzy number-valued function, which are useful for fuzzy optimization.

In this paper, we develop an approach based on the proposed aggregation operators to multiple attribute decision making. Finally, an illustrative example is given to show the developed method. In order to this, the remainder of the paper is structured as follows. Section 2 introduces the basic concepts of fuzzy set, fuzzy number, intuitionistic fuzzy set, intuitionistic fuzzy number, and some operational laws of intuitionistic fuzzy numbers (IFNs). Section 3 presents some aggregate operators with intuitionistic fuzzy information. The novel method for decision making with intuitionistic fuzzy information based on the given operators is 
Fuzzy Information Aggregation in Insurance

introduced in the last part of this section. Section 4 presents a numerical example, and the last section includes some concluding remarks.

\section{Basic concepts}

A crisp set is a regular set and a crisp number is a real number. In fuzzy logic it is allowed for truth values to be any number in the interval $[0,1]$.

Definition 1. (Zadeh, 1965) Let $X$ be a fixed set. Then $F=\left\{\left\langle x, \mu_{F}(x)\right\rangle \mid x \in X\right\}$ is called a fuzzy set, where $\mu_{F}: X \rightarrow[0,1]$ is the membership function of $F$, and $\mu_{F}(x)$ indicates the membership degree of the element $x$ to $F$.

In order to determine the algebra of fuzzy subsets of $X$, we have to specify the intersection and union. Let $A$ and $B$ be two fuzzy sets. Then the intersection is $C=$ $A \cap B$, where the value of $\mu_{C}(x)$ will be a function of the two values $\mu_{A}(x)$ and $\mu_{B}(x)$. This unknown function will be $i(a, b)$, with $a, b \in[0,1]$. Thus, $\mu_{C}(x)=$ $i\left(\mu_{A}(x), \mu_{B}(x)\right)$, for all $x$ in $X$, defines the membership function of $C$. Possible choices for $i(a, b)$ could be $i(a, b)=a b, i(a, b)=\min (a, b)$, $i(a, b)=\sqrt{\max \left(0, a^{2}+b^{2}-1\right)}$.

The functions used for intersection of fuzzy sets are called $t$-norms.

Let $\mathbf{R}$ be the set of all real numbers and $\mathbf{R}^{+}$the set of all positive real numbers.

Definition 2. A $t$-norm $T$ is a function $y=T(a, b)$, where $a, b, y \in[0,1]$, having the following properties:

i) $T(a, 1)=a$;

ii) $T(a, b)=T(b, a)$;

iii) if $b \leq c$, then $T(a, b) \leq T(a, c)$;

iv) $T(a, T(b, c))=T(T(a, b), c)$.

The basic $t$-norms $\operatorname{are} T_{m}(a, b)=\min (a, b), T_{b}(a, b)=\max (0, a+b-1)$, $T_{p}(a, b)=a b, T^{*}(a, b)=\left\{\begin{array}{lc}a, & \text { if } b=1 \\ b, & \text { if } a=1 . \\ 0, & \text { otherwise }\end{array}\right.$.

$T_{m}$ is called standard intersection, $T_{b}$ is bounded sum, $T_{p}$ is algebraic product, and $T^{*}$ is drastic intersection.

\section{Remark 1.}

i) $\quad T^{*}(a, b) \leq T_{b}(a, b) \leq T_{p}(a, b) \leq T_{m}(a, b)$, for all $a$, bin $[0,1]$.

ii) If $T$ is any $t$-norm, then $T^{*}(a, b) \leq T(a, b) \leq T_{m}(a, b)$. 
Let $A$ and $B$ be two fuzzy sets. Then the union is $D=A \cup B$, where the value of $\mu_{D}(x)$ will be a function of the two values $\mu_{A}(x)$ and $\mu_{B}(x)$. Let this function be $u(a, b)$, with $a, b \in[0,1]$. Thus, $\mu_{D}(x)=u\left(\mu_{A}(x), \mu_{B}(x)\right)$, for all $x$ in $X$, defines the membership function of $D$. Choices for $u(a, b)$ could be $u(a, b)=a+$ $b-a b, u(a, b)=\max (a, b)$, and $u(a, b)=\min \left(1, \sqrt{a^{2}+b^{2}}\right)$.

The functions used for union are called $t$-conorms.

Definition 3. A $t$-conorm $U$ is a function $z=U(a, b)$, where $a, b, z \in[0,1]$, having the following properties:

i) $U(a, 0)=a$;

ii) $U(a, b)=U(b, a)$;

iii) if $b \leq c$, then $U(a, b) \leq U(a, c)$;

iv) $U(a, U(b, c))=U(U(a, b), c)$.

The basic $t$-conorms are $U_{m}(a, b)=\max (a, b), U_{b}(a, b)=\min (1, a+b)$, $U_{p}(a, b)=a+b-a b, U^{*}(a, b)=\left\{\begin{array}{cc}a, & \text { if } b=0 \\ b, & \text { if } a=0 . \\ 1, & \text { otherwise }\end{array}\right.$.

$U_{m}$ is called standard union, $U_{b}$ is bounded sum, $U_{p}$ is algebraic sum and $U^{*}$ is drastic union.

\section{Remark 2.}

i) $U_{m}(a, b) \leq U_{p}(a, b) \leq U_{b}(a, b) \leq U^{*}(a, b)$, for all $a$, $b$ in $[0,1]$.

ii) If $U$ is any $t$-conorm, then $U_{m}(a, b) \leq U(a, b) \leq U^{*}(a, b)$.

One of the general union function is Yager's union function defined as: $U_{w}(a, b)=\min \left[1,\left(a^{w}+b^{w}\right)^{\frac{1}{w}}\right]$, where $w \in(0, \infty)$. For instance, $w=1$ leads to $U_{1}(a, b)=\min (1, a+b)$, that is, the bounded sum. For $a, b \in(0,1), a<b$, note that $x(w)=\left(a^{w}+b^{w}\right)^{\frac{1}{w}}$.

We obtain

$$
\begin{aligned}
\lim _{w \rightarrow \infty} \ln x(w) & =\lim _{w \rightarrow \infty} \frac{a^{w} \ln a+b^{w} \ln b}{a^{w}+b^{w}} \\
& =\lim _{w \rightarrow \infty} \frac{(a / b)^{w} \ln a+\ln b}{(a / b)^{w}+1} \\
& =\ln b,
\end{aligned}
$$

thus $\lim _{w \rightarrow \infty} x(b)=b=\max (a, b)$. Similarly, it can be proven for the another case. Therefore $\lim _{w \rightarrow \infty} U_{w}(a, b)=\max (a, b)$.

Proposition 1.Assuming that $w \rightarrow \infty$, Yager union function is transformed into the standard union function. 
In practice one usually uses a pair of $T$ and $U$ which are dual. $T$ and $U$ are dual when $T(a, b)=1-U(1-a, 1-b), U(a, b)=1-T(1-a, 1-b)$. Using the associativity property of $T$ and $U$, we define $T\left(a_{1}, a_{2}, \ldots, a_{n}\right)$ and $U\left(a_{1}, a_{2}, \ldots, a_{n}\right)$, for $a_{i} \in[0,1], 1 \leq i \leq n$, as

$$
\begin{aligned}
& T_{m}\left(a_{1}, a_{2}, \ldots, a_{n}\right)=\min \left(a_{1}, a_{2}, \ldots, a_{n}\right), \\
& U_{m}\left(a_{1}, a_{2}, \ldots, a_{n}\right)=\max \left(a_{1}, a_{2}, \ldots, a_{n}\right), \\
& T_{b}\left(a_{1}, a_{2}, \ldots, a_{n}\right)=\max \left(0, \sum_{i=1}^{n} a_{i}-n+1\right) \\
& U_{b}\left(a_{1}, a_{2}, \ldots, a_{n}\right)=\min \left(1, \sum_{i=1}^{n} a_{i}\right) \\
& T_{p}\left(a_{1}, a_{2}, \ldots, a_{n}\right)=a_{1} a_{2} \cdots a_{n}, \\
& U_{p}\left(a_{1}, a_{2}, \ldots, a_{n}\right)=\sum_{i=1}^{n} a_{i}-\sum_{1 \leq i<j \leq n} a_{i} a_{j} \\
& +\sum_{a \leq i<j<k \leq n} a_{i} a_{j} a_{k}+\ldots+(-1)^{n-1} a_{1} a_{2} \cdots a_{n} .
\end{aligned}
$$

Considering Yager union function, we have

$$
T_{w}(a, b)=1-\min \left[1,\left((1-a)^{w}+(1-b)^{w}\right)^{\frac{1}{w}}\right]
$$

$\operatorname{andlim}_{w \rightarrow \infty} T_{w}(a, b)=\min (a, b)$.

Proposition 2.If $w \rightarrow \infty$, then the function $T_{w}$ converges to the standard intersection function.

The concepts of $\varphi$-normed space and fuzzy $\varphi$-normed space were introduced as generalizations of those of normed space and fuzzy normed space, by considering an appropriate function $\varphi$ in the homogeneity axiom. $\varphi: \mathbf{R} \rightarrow \mathbf{R i s}$ a function with the properties:

i) $\varphi(-t)=\varphi(t)$ for every $t \in \mathbf{R}$,

ii) $\varphi(1)=1$,

iii) $\varphi$ is strictly increasing and continuous on $[0, \infty), \varphi(0)=0$ and $\lim _{t \rightarrow \infty} \varphi(t)=\infty$.

Definition 4. (Atanassov, 1986) An intuitionistic fuzzy set (IFS) is an object

$$
A=\left\{\left\langle x, \mu_{A}(x), v_{A}(x)\right\rangle \mid x \in X\right\}
$$

which is characterized by a membership function $\mu_{A}: X \rightarrow[0,1]$, and a nonmembership function $v_{A}: X \rightarrow[0,1]$ with the condition: $0 \leq \mu_{A}(x)+v_{A}(x) \leq 1$, for all $x \in X$, where $\mu_{A}(x)$ is the membership degree of $x$ in $A$, and $v_{A}(x)$ represent the non-membership degree of $x$ in $A$.

For each IFS $A$ in $X$, if

$$
\pi_{A}(x)=1-\mu_{A}(x)-v_{A}(x), \text { for all } x \in X,
$$


then $\pi_{A}(x)$ is called an indeterminancy degree (hesitancy degree or intuitionistic index) of $x$ in $A$. In particular, if $\pi_{A}(x)=0$, for all $x \in X$, then $A$ reduces to Zadeh's fuzzy set.

For convenience, $\alpha=\left(\mu_{\alpha}, v_{\alpha}\right)$ is an intuitionistic fuzzy number (IFN) or an intuitionistic fuzzy value (IFV), where $\mu_{\alpha} \in[0,1], v_{\alpha} \in[0,1], \mu_{\alpha}+v_{\alpha} \leq 1$. Let us consider $\Theta$ be the set of all IFNs. For any IFN $\alpha=\left(\mu_{\alpha}, v_{\alpha}\right)$, the score of $\alpha$ can be evaluated by the score function $s$ defined as $s(\alpha)=\mu_{\alpha}-v_{\alpha}$, where $s(\alpha) \in$ $[-1,1]$. The larger the score $s(\alpha)$, then the larger the IFN $\alpha$. Clearly, $\alpha^{+}=(1,0)$ is the largest IFN, and $\alpha^{-}=(0,1)$ is the smallest IFN. It is defined an accuracy function $h: h(\alpha)=\mu_{\alpha}+v_{\alpha}$, where $h(\alpha)$ is the accuracy degree of the IFN $\alpha=$ $\left(\mu_{\alpha}, v_{\alpha}\right)$.

\section{Aggregate operators with fuzzy information}

A method for comparison and ranking of two IFNs is based on the score function $s$ and the accuracy function $h$.

Definition 5. Let $\alpha_{1}=\left(\mu_{\alpha_{1}}, v_{\alpha_{1}}\right)$ and $\alpha_{2}=\left(\mu_{\alpha_{2}}, v_{\alpha_{2}}\right)$ be two IFNs, the score function $s$ and the accuracy function $h$. Then:

- If $s\left(\alpha_{1}\right)<s\left(\alpha_{2}\right)$, then the IFN $\alpha_{1}$ is smaller than the IFN $\alpha_{2}$, denoted $\alpha_{1}<\alpha_{2}$.

- If $s\left(\alpha_{1}\right)=s\left(\alpha_{2}\right)$, then

1) If $h\left(\alpha_{1}\right)=h\left(\alpha_{2}\right)$, the IFNs $\alpha_{1}$ and $\alpha_{2}$ are equal, denoted $\alpha_{1}=\alpha_{2}$;

2) If $h\left(\alpha_{1}\right)<h\left(\alpha_{2}\right)$, the IFN $\alpha_{1}$ is smaller than the IFN $\alpha_{2}$, denoted $\alpha_{1}<\alpha_{2}$;

3) If $h\left(\alpha_{1}\right)>h\left(\alpha_{2}\right)$, the IFN $\alpha_{1}$ is larger than the IFN $\alpha_{2}$, denoted $\alpha_{1}>\alpha_{2}$.

- If $s\left(\alpha_{1}\right)>s\left(\alpha_{2}\right)$, then the IFN $\alpha_{1}$ is larger than the IFN $\alpha_{2}$, denoted $\alpha_{1}>\alpha_{2}$.

An interesting research topic is "how to aggregate a collection of IFNs without any loss of information".

Definition 6.Let $a_{j}, j=1,2, \ldots, n$, be a collection of real numbers, $\omega=$ $\left(\omega_{1}, \omega_{2}, \ldots, \omega_{n}\right)$ is the weight vector of $a_{j}$ numbers, with $\omega_{j} \in[0,1], j=1,2, \ldots, n$, and $\sum_{j=1}^{n} \omega_{j}=1$, and $W A: \mathbf{R}^{n} \rightarrow \mathbf{R}$. If $W A_{\omega}\left(a_{1}, a_{2}, \ldots, a_{n}\right)=\sum_{j=1}^{n} \omega_{j} \cdot a_{j}$, then the function $W A$ is called a weighted averaging operator.

Definition 7. Let $W G_{\omega}:\left(\mathbf{R}^{+}\right)^{n} \rightarrow \mathbf{R}^{+}$. If $W G_{\omega}\left(a_{1}, a_{2}, \ldots, a_{n}\right)=\prod_{j=1}^{n} a_{j}^{\omega_{j}}$, then the function $W G$ is called a weighted geometric operator, where $\omega=$ $\left(\omega_{1}, \omega_{2}, \ldots, \omega_{n}\right)$ is the exponential weighting vector of $a_{j}$ numbers, with $\omega_{j} \in$ $[0,1], j=1,2, \ldots, n$, and $\sum_{j=1}^{n} \omega_{j}=1$.

The difference between these two operators is that the $W G$ operator is much more sensitive to the given arguments. Especially in the case where there is an 
Fuzzy Information Aggregation in Insurance

argument taking the value of zero, the aggregated value of these arguments by using the $W G$ operator must be zero no matter what the other given arguments are.

Definition 8. Let $O W A: \mathbf{R}^{n} \rightarrow \mathbf{R}$. If $O W A_{\omega}\left(a_{1}, a_{2}, \ldots, a_{n}\right)=\sum_{j=1}^{n} \omega_{j} \cdot b_{j}$, where $\omega=\left(\omega_{1}, \omega_{2}, \ldots, \omega_{n}\right)$ is the weighting vector associated with the function $O W A$, with $\omega_{j} \in[0,1], j=1,2, \ldots, n$, and $\sum_{j=1}^{n} \omega_{j}=1$, and $b_{j}$ is the $j$-th largest of $a_{j}, j=$ $1,2, \cdots, n$, then the function $O W A$ is called an ordered weighted averaging operator.

Definition 9.Let $O W G:\left(\mathbf{R}^{+}\right)^{n} \rightarrow \mathbf{R}^{+}$. If $O W G_{w}\left(a_{1}, a_{2}, \ldots, a_{n}\right)=\prod_{j=1}^{n} b_{j}^{w_{j}}$, where $w=\left(w_{1}, w_{2}, \ldots, w_{n}\right)$ is the exponential weighting vector associated with the function $O W G$, with $\omega_{j} \in[0,1], j=1,2, \ldots, n, \sum_{j=1}^{n} \omega_{j}=1$, and $b_{j}$ is the $j$-th largest of $a_{j}, j=1,2, \ldots, n$, then the function $O W G$ is called an ordered weighted geometric operator.

Definition 10.Let $\alpha_{i}=\left(\mu_{\alpha_{i}}, v_{\alpha_{i}}\right), i=1,2, \cdots, n$, a collection of intuitionistic fuzzy numbers, and $\omega=\left(\omega_{1}, \omega_{2}, \ldots, \omega_{n}\right)$ the weight vector of $\alpha_{i}, i=1,2, \ldots, n$, with $\omega_{i} \in[0,1], i=1,2, \ldots, n$, and $\sum_{i=1}^{n} \omega_{i}=1$, then $\operatorname{IFWA}_{\omega}\left(\alpha_{1}, \alpha_{2}, \ldots, \alpha_{n}\right)=$ $\omega_{1} \alpha_{1} \oplus \omega_{2} \alpha_{2} \oplus \cdots \oplus \omega_{n} \alpha_{n}$ is called an intuitionistic fuzzy weighted averaging operator.

Definition 11. Let $p, q \geq 0, p+q>0$, and $\alpha_{i} \geq 0, i=1,2, \cdots, n$, a collection of non-negative real numbers, then $B^{p, q}\left(\alpha_{1}, \alpha_{2}, \ldots, \alpha_{n}\right)=\left(\frac{1}{n \cdot(n-1)} \sum_{\substack{i, j=1 \\ i \neq j}}^{n} \alpha_{i}^{p} \cdot \alpha_{j}^{q}\right)^{\frac{1}{p+q}}$ is called the Bonferroni mean (BM).

Definition 12. Let $p, q \geq 0, p+q>0$, and $\alpha_{i}, i=1,2, \ldots, n$, a collection of intuitionistic fuzzy numbers, and $\omega=\left(\omega_{1}, \omega_{2}, \ldots, \omega_{n}\right)$ the weight vector of $\alpha_{i}, i=$ $1,2, \ldots, n$, where $\omega_{i}$ indicates the importance degree of $\alpha_{i}$, satisfying $\omega_{i} \in$ $[0,1], i=1,2, \ldots, n$, and $\sum_{i=1}^{n} \omega_{i}=1$, then

$\operatorname{IFB}_{\omega}^{p, q}\left(\alpha_{1}, \alpha_{2}, \cdots, \alpha_{n}\right)=\left(\frac{1}{n \cdot(n-1)}\left(\prod_{\substack{i, j=1 \\ i \neq j}}^{n}\left(\left(\omega_{i} \alpha_{i}\right)^{p} \otimes\left(\omega_{j} \alpha_{j}\right)^{q}\right)\right)\right)^{\frac{1}{p+q}}$ is called the weighted intuitionistic fuzzy Bonferroni mean (WIFBM).

Theorem 1. (Xu and Yager, 2011) The aggregated value using the WIFBM is an IFN, and 
Mihaela Covrig, Paul Tanasescu, Iulian Mircea

$$
\begin{aligned}
& \operatorname{WIFB}_{\omega}^{p, q}\left(\alpha_{1}, \alpha_{2}, \cdots, \alpha_{n}\right)= \\
= & \left(\left(1-\prod_{\substack{i, j=1 \\
i \neq j}}^{n}\left(1-\left(1-\left(1-\mu_{\alpha_{i}}\right)^{\omega_{i}}\right)^{p}\left(1-\left(1-\mu_{\alpha_{j}}\right)^{\omega_{j}}\right)^{q}\right)^{m}\right)^{t},\right. \\
& \left.1-\left(1-\prod_{\substack{i, j=1 \\
i \neq j}}^{n}\left(1-\left(1-v_{\alpha_{i}}^{\omega_{i}}\right)^{p}\left(1-v_{\alpha_{j}}^{\omega_{j}}\right)^{q}\right)^{m}\right)^{t}\right),
\end{aligned}
$$

where $m=\frac{1}{n \cdot(n-1)}, t=\frac{1}{p+q}$.

Proposition 3. If $\alpha=\left(\mu_{\alpha}, v_{\alpha}\right), \alpha_{1}=\left(\mu_{\alpha_{1}}, v_{\alpha_{1}}\right)$, and $\alpha_{2}=\left(\mu_{\alpha_{2}}, v_{\alpha_{2}}\right)$ are IFNs, then

1) $\bar{\alpha}=\left(v_{\alpha}, \mu_{\alpha}\right)$;

2) $\alpha_{1} \wedge \alpha_{2}=\left(\min \left\{\mu_{\alpha_{1}}, \mu_{\alpha_{2}}\right\}, \max \left\{v_{\alpha_{1}}, v_{\alpha_{2}}\right\}\right)$;

3) $\alpha_{1} \vee \alpha_{2}=\left(\max \left\{\mu_{\alpha_{1}}, \mu_{\alpha_{2}}\right\}, \min \left\{v_{\alpha_{1}}, v_{\alpha_{2}}\right\}\right)$;

4) $\alpha_{1} \oplus \alpha_{2}=\left(\mu_{\alpha_{1}}+\mu_{\alpha_{2}}-\mu_{\alpha_{1}} \cdot \mu_{\alpha_{2}}, v_{\alpha_{1}} \cdot v_{\alpha_{2}}\right)$;

5) $\alpha_{1} \otimes \alpha_{2}=\left(\mu_{\alpha_{1}} \cdot \mu_{\alpha_{2}}, v_{\alpha_{1}}+v_{\alpha_{2}}-v_{\alpha_{1}} \cdot v_{\alpha_{2}}\right)$;

6) $\lambda \alpha=\left(1-\left(1-\mu_{\alpha}\right)^{\lambda}, v_{\alpha}^{\lambda}\right), \lambda>0$;

7) $\alpha^{\lambda}=\left(\mu_{\alpha}^{\lambda}, 1-\left(1-v_{\alpha}\right)^{\lambda}\right), \lambda>0$,

are IFNs.

For a multi-attribute decision making problem, let $Y=\left\{Y_{1}, Y_{2}, \ldots, Y_{n}\right\}$ be a finite set of alternatives, $G=\left\{G_{1}, G_{2}, \ldots, G_{m}\right\}$ a set of attributes, and $\omega=$ $\left(\omega_{1}, \omega_{2}, \ldots, \omega_{m}\right)$ the weight vector of attributes, where $\omega_{j} \in[0,1], j=1,2, \ldots, m$, and $\sum_{j=1}^{m} \omega_{j}=1$. Suppose that the characteristics of the alternatives $Y_{i}, i=$ $1,2, \ldots, n$, are represented by the IFNs. For convenience, let $\rho_{i j}=\left(t_{i j}, f_{i j}\right)$ denote the characteristic of the alternative $Y_{i}$ with respect to the attribute $G_{j}$, where $t_{i j}$ indicates the degree that the alternative $Y_{i}$ satisfies the attribute $G_{j}$, and $f_{i j}$ indicates the degree that the alternative $Y_{i}$ does not satisfy the attribute $G_{j}$. Therefore, the characteristics can be contained in an intuitionistic fuzzy decision matrix $\rho=$ $\left(\rho_{i j}\right)_{n \times m}$, where $t_{i j} \in[0,1], f_{i j} \in[0,1]$, and $t_{i j}+f_{i j} \leq 1$. If all the attributes $G_{j}, j=1,2, \ldots, m$, are of the same type, then the attribute values do not need normalization. However, there are generally benefit attributes and cost attributes in multi-attribute decision making. In such cases, we transform the attribute values of cost type into the attribute values of benefit type, then the matrix $\rho=\left(\rho_{i j}\right)_{n \times m}$ 
can be transformed into the intuitionistic fuzzy decision matrix $R=\left(r_{i j}\right)_{n \times m}$, where

$$
r_{i j}=\left(\mu_{i j}, v_{i j}\right)=\left\{\begin{array}{ll}
\rho_{i j}, & \text { for benefit attribute } G_{j}, \\
\bar{\rho}_{i j}, & \text { for cost attribute } G_{j}
\end{array},\right.
$$

where $\bar{\rho}_{i j}$ is the complement of $\rho_{i j}$, and therefore $\bar{\rho}_{i j}=\left(f_{i j}, t_{i j}\right)$.

The proposed approach to multi-attribute decision making with intuitionistic fuzzy information involves the following steps:

Step 1: Let $Y, G, \omega$ and matrix $\rho$ be defined as before. If it is necessary, we can transform $\rho$ into the normalized intuitionistic fuzzy decision matrix $R$.

Step 2: Utilize the WIFBM and IFWA operators to aggregate all the characteristics of the $i$-th line, and calculate the overall attribute values $r b_{i}, r a_{i}$ corresponding to the alternative $Y_{i}, i=1,2, \ldots, n$.

Step 3: Based on Definition 5, rank all the alternatives $Y_{i}, i=1,2, \ldots, n$, in accordance with values $r b_{i}$, and respectively $r a_{i}, i=1,2, \ldots, n$. Calculate, as well, the average scores for the mixed method, that is, the average of the scores obtained using the operators WIFBM and IFWA.

Step 4: Rank all the alternatives in ascending order, and then select the most desirable alternative.

\section{Numerical illustration and conclusions}

Example. We consider a complex insurance product offered by four insurers, $Y_{i}, i=1,2,3,4$. Suppose that there are three attributes: $G_{1}$ : the(economic) cost, $G_{2}$ : insurance coverage degree, $G_{3}$ : credibility of insurer. The weight vector $\omega$ of the attributes is $\omega=(0.3 ; 0.3 ; 0.4)$. The characteristics of the alternatives $Y_{i}$ are represented by the IFNs, as shown in Table 1 .

Table 1. The IFNs attributes $G_{1}, G_{2}, G_{3}$ for the insurers $Y_{i}, i=1,2,3,4$.

\begin{tabular}{|c|c|c|c|}
\hline & $G_{1}$ & $G_{2}$ & $G_{3}$ \\
\hline$Y_{1}$ & $(0.3 ; 0.6)$ & $(0.4 ; 0.5)$ & $(0.7 ; 0.2)$ \\
\hline$Y_{2}$ & $(0.3 ; 0.5)$ & $(0.7 ; 0.2)$ & $(0.4 ; 0.3)$ \\
\hline$Y_{3}$ & $(0.5 ; 0.4)$ & $(0.6 ; 0.4)$ & $(0.5 ; 0.3)$ \\
\hline$Y_{4}$ & $(0.3 ; 0.7)$ & $(0.5 ; 0.4)$ & $(0.3 ; 0.6)$ \\
\hline
\end{tabular}

We calculated the weighted intuitionistic fuzzy Bonferroni means, they are given below:

$$
\begin{aligned}
& \operatorname{WIFBM}_{\omega}^{1,1}\left(Y_{1}\right)=(0.245784 ; 0.687961), \\
& \operatorname{WIFBM}_{\omega}^{1,1}\left(Y_{2}\right)=(0.221964 ; 0.344025),
\end{aligned}
$$


Mihaela Covrig, Paul Tanasescu, Iulian Mircea

$$
\begin{aligned}
& \operatorname{WIFBM}_{\omega}^{1,1}\left(Y_{3}\right)=(0.205709 ; 0.735848), \\
& \operatorname{WIFBM}_{\omega}^{1,1}\left(Y_{4}\right)=(0.202034 ; 0.759500) .
\end{aligned}
$$

Therefore, the ranking is $Y_{4} \prec Y_{3} \prec Y_{1} \prec Y_{2}$, where " $\prec$ " denotes "be inferior to", and according to the order on Bonferroni means, the best alternative is $Y_{2}$.

We calculated the intuitionistic fuzzy weighted averages, they are presented below:

$$
\begin{aligned}
& I F W A_{\omega}\left(Y_{1}\right)=(0.59736467 ; 0.29733044), \\
& I F W A_{\omega}\left(Y_{2}\right)=(0.53858907 ; 0.26564024), \\
& I F W A_{\omega}\left(Y_{3}\right)=(0.50608595 ; 0.38120404), \\
& I F W A_{\omega}\left(Y_{4}\right)=(0.50924289 ; 0.43153385) .
\end{aligned}
$$

Therefore, the ranking is $Y_{4} \prec_{a} Y_{3} \prec_{a} Y_{2} \prec_{a} Y_{1}$, where " $\prec_{a}$ " denotes "be inferior to", and according to the order on weighted averaging, the best alternative is $Y_{1}$.

Using the new method, called the mixt method, the third one, we have the next score functions:

$$
\begin{aligned}
& \operatorname{sm}\left(Y_{1}\right)=-0.07107123, \\
& \operatorname{sm}\left(Y_{2}\right)=0.07544391, \\
& \operatorname{sm}\left(Y_{3}\right)=-0.20262805, \\
& \operatorname{sm}\left(Y_{4}\right)=-0.23987852 .
\end{aligned}
$$

Therefore, the ranking is $Y_{4} \prec_{m a} Y_{3} \prec_{m a} Y_{1} \prec_{m a} Y_{2}$, where " $\prec_{m a}$ " denotes "be inferior to", thus according to the order of the mixed method, the best alternative is $Y_{2}$. 


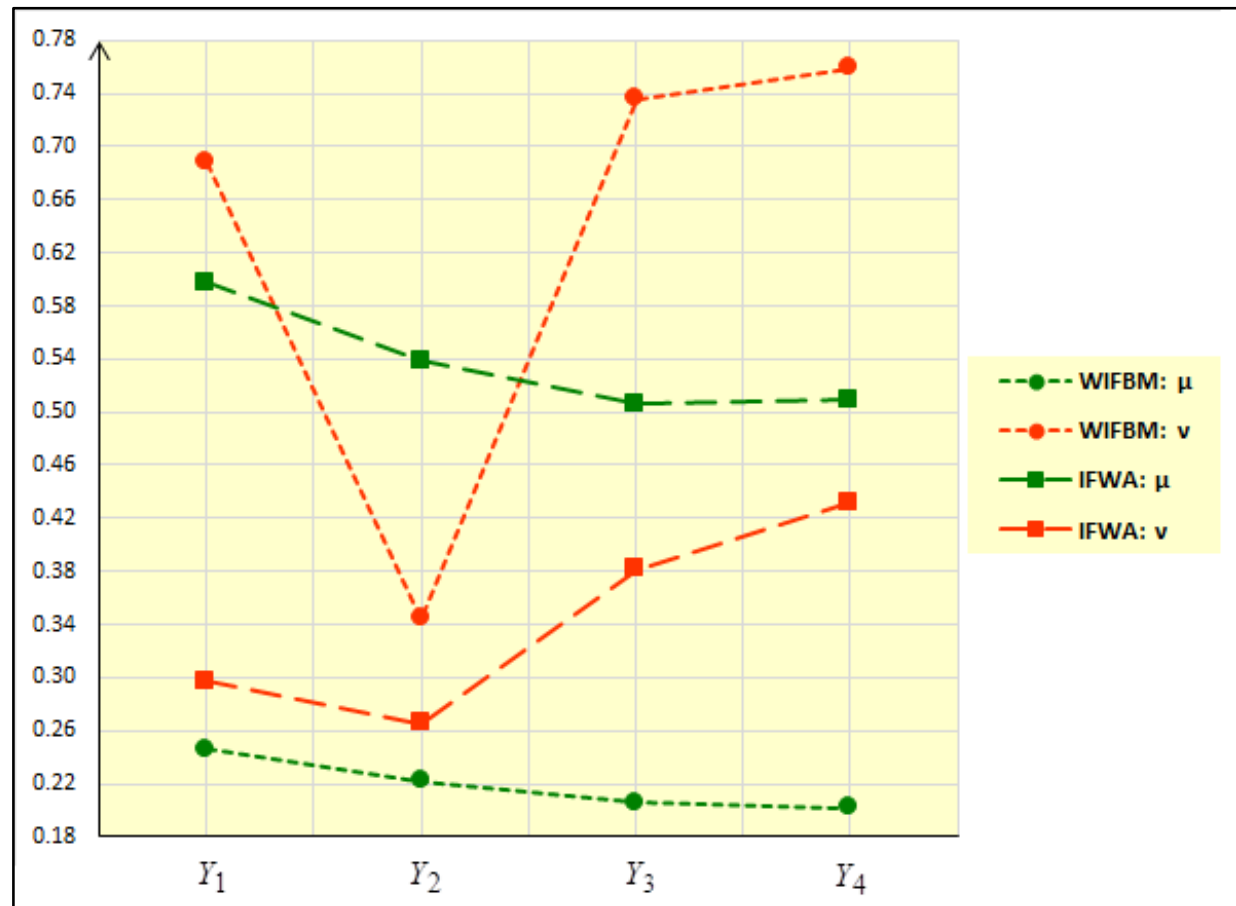

Figure 1. The values of the membership and non-membership functions.

Of the three rankings, two indicate the insurer $Y_{2}$ as the best choice. We suggest the use of the three methods for choosing the suitable alternative, in the case of our example, the insurer.

So far we considered both the membership and non-membership degree. Let us suppose we only take into account the membership degree, all the other information in the example being unchanged. Table 2 presents the fuzzy numbers attributes $G_{1}, G_{2}, G_{3}$ for the insurers $Y_{1}, Y_{2}, Y_{3}, Y_{4}$. The last two attributes can be considered as benefits for the possible client, while the first one is perceived as a cost.

Table 2. The FNs attributes $G_{1}, G_{2}, G_{3}$ for the insurers $Y_{i}, i=1,2,3,4$.

\begin{tabular}{|c|c|c|c|}
\hline & $G_{1}$ & $G_{2}$ & $G_{3}$ \\
\hline$Y_{1}$ & 0.6 & 0.4 & 0.7 \\
\hline$Y_{2}$ & 0.5 & 0.7 & 0.4 \\
\hline$Y_{3}$ & 0.4 & 0.6 & 0.5 \\
\hline$Y_{4}$ & 0.7 & 0.5 & 0.3 \\
\hline
\end{tabular}


If we work with the characteristics of the alternatives $Y_{i}$ represented by the fuzzy numbers (FNs), as shown in Table 2, we obtain the following results.

The weighted fuzzy Bonferroni means are given below

$$
\begin{aligned}
& W F B M_{\omega}^{1,1}\left(Y_{1}\right)=0.24578406 \\
& W F B M_{\omega}^{1,1}\left(Y_{2}\right)=0.221964173 \\
& W F B M_{\omega}^{1,1}\left(Y_{3}\right)=0.205710007 \\
& W F B M_{\omega}^{1,1}\left(Y_{4}\right)=0.202034018 .
\end{aligned}
$$

Therefore $Y_{4} \prec Y_{3} \prec Y_{2} \prec Y_{1}$, thus, according to the order on Bonferroni means, the best alternative is $Y_{1}$.

We calculated the fuzzy weighted averages:

$$
\begin{aligned}
& F W A_{\omega}\left(Y_{1}\right)=0.597364672, \\
& F W A_{\omega}\left(Y_{2}\right)=0.538589069, \\
& F W A_{\omega}\left(Y_{3}\right)=0.506085957, \\
& F W A_{\omega}\left(Y_{4}\right)=0.509242897 .
\end{aligned}
$$

Therefore $Y_{3} \prec_{a} Y_{4} \prec_{a} Y_{2} \prec_{a} Y_{1}$, thus, according to the order on weighted average, the best alternative is $Y_{1}$.

Using the mixt method we calculated the following score functions:

$$
\begin{aligned}
& \operatorname{sm}\left(Y_{1}\right)=0.421574366, \\
& \operatorname{sm}\left(Y_{2}\right)=0.380276621, \\
& \operatorname{sm}\left(Y_{3}\right)=0.355897982, \\
& \operatorname{sm}\left(Y_{4}\right)=0.355638457 .
\end{aligned}
$$

Therefore $Y_{4} \prec_{m a} Y_{3} \prec_{m a} Y_{2} \prec_{m a} Y_{1}$, thus according to the order of the mixed method, the best alternative is $Y_{1}$.

The three rankings indicate the insurer $Y_{1}$ as the best choice. With respect to the first part of the example, the one in which we have considered the intuitionistic fuzzy numbers, the choice of another insurer in the second case could be explained by the smaller amount of information provided in Table 2 .

\section{Conclusion}

The main purpose of the present paper was to develop a new fuzzy information aggregation procedure which is based on a weighted intuitionistic fuzzy Bonferroni mean and an aggregation procedure introduced by Xu and Yager in 2011.

This new method brings new insights in solving real-life problems insurance companies may face while acting in a competitive market, where they want to be selected by clients as the best alternative. Such situations can be modeled by a multi-attribute decision making and selection of the insurance product or the insurance company.

The illustrative example was designed to give a suggestive real-life situation of four competing insurance companies offering a specific insurance product. Possible clients have information on three attributes of those insurers: one is 
Fuzzy Information Aggregation in Insurance

related to the economic cost, and the other two to some benefits granted. The results show that, considering fuzzy numbers attributes, all procedures indicated the same insurer as best alternative, while considering intuitionistic fuzzy numbers attributes, the three procedures yielded different rankings. The additional information brought by the non-membership degree to the intuitionistic fuzzy numbers is the explanation to such a situation. Therefore, the proposed procedure could be another instrument in the evaluation of the possible best alternatives as it provides more refined alternatives for an insurance company.

ACKNOWLEDGEMENT. This work has been presented at FIBA 2018 Conference, held at The Bucharest University of Economics, on March29-30, 2018. The authors would like to thank to all those who attended the presentation for the valuable suggestions and insights. They came both from the participants as well as from the members of the Department of Finance. In the final version of the paper we took into consideration those comments.

\section{REFERENCES}

[1] Atanassov, K. (1986), Intuitionistic Fuzzy Sets. Fuzzy Sets and Systems, 20(1), 87-96;

[2] Bonferroni, C. (1950), Sullemedie multiple di potenze. Bolletinodell'Unione Matematicaltaliana, 5(3-4), 267-270;

[3] Carlsson, C., Fullér, R. (2003), A Fuzzy Approach to Real Option Valuation. Fuzzy Sets and Systems, 139(2), 297-312;

[4] Chai, Y., Zhang, D. (2016), A Representation of Fuzzy Numbers. Fuzzy Sets and Systems, 295, 1-18;

[5] Chalco-Cano, Y., Lodwick, W.A., Bede, B. (2014), Single Level Constraint Interval Arithmetic; Fuzzy Sets and Systems, 257, 146-168;

[6] Chen, N., Xu, Z. S., Xia, M. (2013), Correlation Coefficients of Hesitant Fuzzy Sets and their Applications to Clustering Analysis. Applied Mathematical Modelling, 37(4), 2197-2211;

[7] Chen, S.L., Li, J.G., Wang, X.G. (2005), Fuzzy Sets Theory and Applications; Science Press, Beijing;

[8] Chiu, C. Y., Park, C. S. (1994), Fuzzy Cash Flow Analysis Using Present Worth Criterion. The Engineering Economist, 39(2), 113-138;

[9] Delgado, M., Vila, A., Voxman, W. (1998), On a Canonical Representation of Fuzzy Numbers. Fuzzy Sets and Systems, 93(1), 125-135;

[10] Gong, Z., Hai, S. (2016), Convexity of n-dimensional Fuzzy Numbervalued Functions and its Applications. Fuzzy Sets and Systems, 295, 19-36;

[11] Miheț, D., Zaharia, C. (2014), A Note on the Definition of a Generalized Fuzzy Normed Space. Fuzzy Sets and Systems, 238, 129-134; 
Mihaela Covrig, Paul Tanasescu, Iulian Mircea

[12] Moon, K. S., Kim, H. (2013), A Multi-dimensional Local Average Lattice Method for Multi-asset Models. Quantitative Finance, 13(6), 873-884;

[13] Torra, V. (2010), Hesitant Fuzzy Sets. International Journal of Intelligent Systems, 25(6), 529-539;

[14] Torra, V. (2003), Information Fusion in Data Mining; Springer, Berlin;

[15] Xia, M., Xu, Z. S. (2011), Hesitant Fuzzy Information Aggregation in Decision Making. International Journal of Approximate Reasoning, 52(3), 395-407;

[16] Xu, Z., Xia, M. (2011), Induced Generalized Intuitionistic Fuzzy Operators. Knowledge-Based Systems, 24(2), 197-209;

[17] Xu, Z., Cai, X. (2012), Intuitionistic Fuzzy Information Aggregation: Theory and Applications; Science Press, Beijing;

[18] Xu, Z., Yager, R.R. (2011), Intuitionistic Fuzzy Bonferroni Means. IEEE Transactions on Systems, Man and Cybernetics, Part B, 41(2), 568-578;

[19] Zadeh, L. (1965), Fuzzy Sets. Information and control, 8(3), 338-353;

[20] Zeng, S. Z., Su, W. H. (2012), Linguistic Induced Generalized Aggregation Distance Operators and their Application to Decision Making. Economic Computation and Economic Cybernetics Studies and Research,46(2), ASE Publishing, 155-172. 